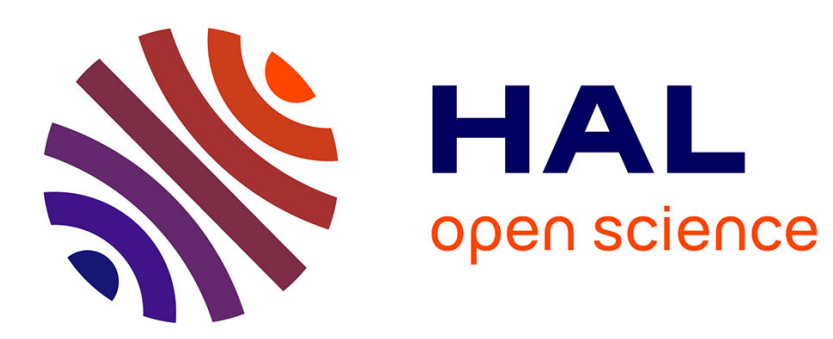

\title{
Voltage support by optimal integration of Plug-in Hybrid Electric Vehicles to a Residential Grid
}

\author{
Andres Ovalle, Seddik Bacha, Ahmad Hably
}

\section{To cite this version:}

Andres Ovalle, Seddik Bacha, Ahmad Hably. Voltage support by optimal integration of Plug-in Hybrid Electric Vehicles to a Residential Grid. IECON 2014 - 40th Annual Conference of the IEEE Industrial Electronics Society, Oct 2014, Dallas, United States. pp.1-6. hal-01064279

\section{HAL Id: hal-01064279 \\ https://hal.science/hal-01064279}

Submitted on 17 Sep 2014

HAL is a multi-disciplinary open access archive for the deposit and dissemination of scientific research documents, whether they are published or not. The documents may come from teaching and research institutions in France or abroad, or from public or private research centers.
L'archive ouverte pluridisciplinaire HAL, est destinée au dépôt et à la diffusion de documents scientifiques de niveau recherche, publiés ou non, émanant des établissements d'enseignement et de recherche français ou étrangers, des laboratoires publics ou privés. 


\section{Voltage support by Optimal integration of Plug-in Hybrid Electric Vehicles to a Residential Grid}

\author{
Andres Ovalle, Seddik Bacha \\ Grenoble Electrical Engineering Laboratory (G2ELab) \\ 38042 Saint Martin d'Hères, France \\ andres.ovalle-villamil@g2elab.grenoble-inp.fr \\ seddik.bacha@g2elab.grenoble-inp.fr
}

\author{
Ahmad Hably \\ Grenoble Image, Speech, Signal and \\ Automation Laboratory (GIPSA-Lab) \\ 38042 Saint Martin d'Hères, France \\ ahmad.hably@gipsa-lab.grenoble-inp.fr
}

\begin{abstract}
This paper provides a linear approach to compute the voltages at any node on a residential grid based on the house instantaneous load and the presence of charging PlugIn Hybrid Electric Vehicles (PHEV) on the grid (and the corresponding instantaneous consumption or injection). Based on this linear operation, the paper provides a detailed linear programming formulation of the problem of charging the PHEVs while providing a voltage support service to the grid. Multiple evaluation cases are included in order to test the ability of the approach to maintain voltages within safety limits and provide optimal consumption/injection policies. An additional case is included to prove the potential of the PHEVs to solve existing voltage technical issues on a residential grid.
\end{abstract}

Index Terms-Plug-In Hybrid Vehicle, Smart charging, Vehicle-to-home, Linear programming, Voltage Support.

\section{INTRODUCTION}

The high level of fossil fuel consumption and the environmental issues caused by conventional vehicles have impulsed the development of several vehicle alternatives. Among those alternatives, Electrical Vehicles (EV) (with the current battery technologies) have been relegated to a second place because of the reduced autonomy compared with conventional vehicles [1]. Hybrid Vehicles (HV) and PHEVs are some of the most applicable solutions available in the automotive industry. HVs employ an energy storage capacity to reduce fuel consumption during strong accelerations. Compared to HVs, PHEVs have a larger energy storage capacity which provides the capacity of working on electrical mode (zero $\mathrm{CO}_{2}$ emissions) with certain autonomy. Also PHEVS can work in a similar mode of HVs [1], [2].

Without a dedicated infrastructure, PHEVs are expected to be charged at homes, representing a significant amount of new load to the residential grids [2], [3]. This increment on the energy demand causes multiple problems and endangers the grid safe operation [4]. The availability of bidirectional chargers can represent a valuable solution to the integration of PHEVs to the grid and the solution of the resulting technical issues [3], [4], [5]. In this way, PHEVs and EVs with improved energy storage systems represent not only loads but mobile energy sources [5].

Appropriate strategies to provide optimal policies of consumption/injection of energy become very crucial for the integration of PHEVs to the grid [2], [3], [4], [5], [6]. This paper provides a linear approach to compute the voltages at any node of a residential test grid based on the house instantaneous load and the presence of PHEVs in the house (and the corresponding instantaneous consumption or injection). Based on this linear operation, the paper provides a detailed linear programming formulation of the problem of charging the PHEVs while providing a voltage support service to the grid. The set of restrictions is established based on the one proposed by [7], [8]. Additionally, multiple evaluation cases are included to test the strategy.

The paper is organized as follows. Section II introduces the modeling approach followed. Section III presents the set of restrictions for the optimization problem. Section IV presents the standard form for the linear programming problem. Section $\mathrm{V}$ presents the study cases and the obtained results. In section VI the paper is concluded.

\section{Modeling of the Grid and the State of Charge (SOC) OF BATTERIES}

The Residential grid is modeled as a radial topology grid with a feeder and branches beginning at certain nodes of the feeder as it is shown on Fig. 1. Because it is a low voltage grid $(R>>X)$, the reactive portion of the lines is neglected. Each node of the grid is considered as a house and each house is able to have one PHEV connected. The load is modeled as a unity power factor load (a resistance) and a current source representing the battery from the PHEV. The resistive load value is approximated with the nominal voltage of the grid and the corresponding instantaneous active power demand from the house, as $R \approx V_{\text {nom }}^{2} / P_{\text {load }}$. The current injected or consumed by the batteries is approximated from the desired instantaneous input/output power value and the nominal voltage as $i_{P H E V} \approx P_{P H E V} / V_{\text {nom }}$. The transformer node is represented as a voltage source with a tension that can be fixed at each time step. The nominal voltage is the transformer voltage and it is common to every node. Reactive power control, unbalances or harmonic can be considered but are not included in this paper. With these assumptions, the grid is modeled as a linear circuit where instantaneous voltages at each node can be computed from the voltage source (the transformer) and the current sources (the PHEVs) at each time step by a linear operation (a matrix multiplication). 

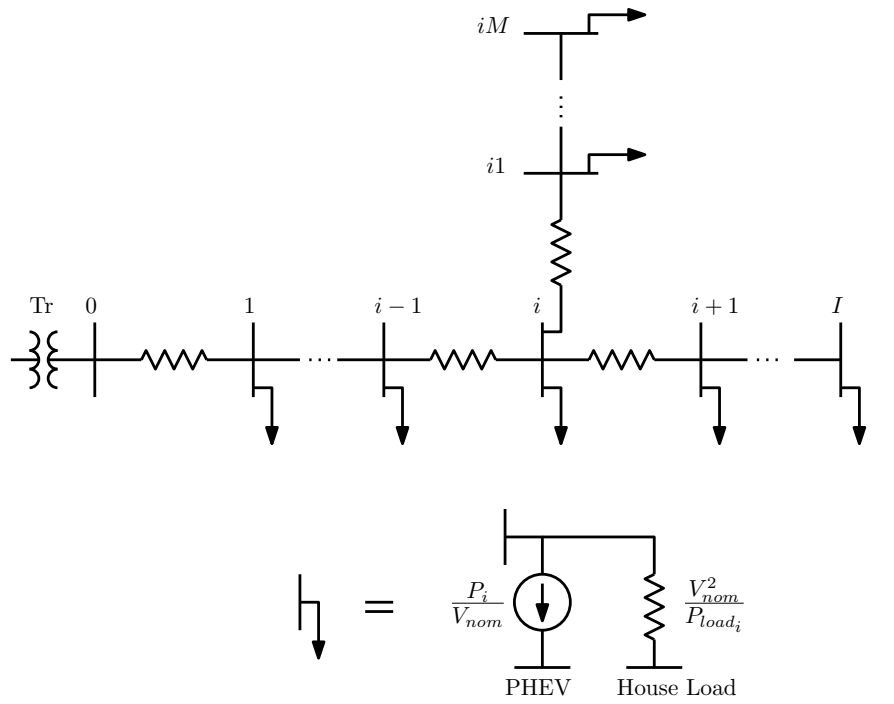

Fig. 1. Radial Grid Modeling

\section{A. Problem Formulation}

The vector of voltages $\mathbf{v}_{t_{k}, n}$ at a given instant $t_{k}$ and at each node $n$ can be computed depending on the load of each home at that instant, and on the power consumed or injected by the PHEVs $\mathbf{p}_{t_{k}, n}$. The vectors of voltage and power of PHEVs are organized as follows.

$$
\begin{gathered}
\mathbf{v}_{t_{k}, n}=\left[v_{t_{k}, 1}, v_{t_{k}, 2}, \cdots, v_{t_{k}, N}\right]^{\mathrm{T}} \\
\mathbf{p}_{t_{k}, n}=\left[p_{t_{k}, 1}, p_{t_{k}, 2}, \cdots, p_{t_{k}, N}\right]^{\mathrm{T}} \\
\mathbf{v}_{t_{k}, n}=\hat{\mathbf{A}}_{t_{k}} \hat{\mathbf{h}}_{t_{k}}+\hat{\mathbf{A}}_{t_{k}} \mathbf{p}_{t_{k}, n}
\end{gathered}
$$

The information of home loads at the time instant $t_{k}$ and at each node is summarized in matrix $\hat{\mathbf{A}}_{t_{k}}$. The expression (1) is employed to compute the vector of voltages $\mathbf{v}_{t_{k}, n}$. The first element of vector $\hat{\mathbf{h}}_{t_{k}}$ has information about the voltage at the transformer node (node 0 ) at time $t_{k}$. This expression is a superposition of two terms. The first term corresponds to the voltage levels contributed by the transformer node. The second term corresponds to the voltage levels contributed by the PHEVs.

In order to compute the voltages at any node for a discretized time frame, the following procedure is proposed.

$$
\begin{aligned}
& \hat{\mathbf{A}}=\left[\begin{array}{cccc}
\hat{\mathbf{A}}_{t_{1}} & \mathbf{0} & \cdots & \mathbf{0} \\
\mathbf{0} & \hat{\mathbf{A}}_{t_{2}} & & \vdots \\
\vdots & & \ddots & \mathbf{0} \\
\mathbf{0} & \cdots & \mathbf{0} & \hat{\mathbf{A}}_{t_{f}}
\end{array}\right] \\
& \mathbf{v}_{t, n}^{\mathrm{T}}=\left[\mathbf{v}_{t_{1}, n}^{\mathrm{T}}, \mathbf{v}_{t_{2}, n}^{\mathrm{T}}, \cdots, \mathbf{v}_{t_{f}, n}^{\mathrm{T}}\right] \\
& \mathbf{p}_{t, n}^{\mathrm{T}}=\left[\mathbf{p}_{t_{1}, n}^{\mathrm{T}}, \mathbf{p}_{t_{2}, n}^{\mathrm{T}}, \cdots, \mathbf{p}_{t_{f}, n}^{\mathrm{T}}\right] \\
& \mathbf{v}_{t, n}=\hat{\mathbf{A}} \hat{\mathbf{h}}_{t}+\hat{\mathbf{A}} \mathbf{p}_{t, n}
\end{aligned}
$$

The block diagonal matrix $\hat{\mathbf{A}}$ can be constructed with matrices $\hat{\mathbf{A}}_{t_{k}}$ corresponding to instants $k=\{1,2, \cdots, f\}$ and the corresponding instantaneous load of each home in that time frame. The vector $\mathbf{v}_{t, n}$ of voltages at any instant of that time frame and at any node is a concatenation of vectors $\mathbf{v}_{t_{k}, n}$ as its is shown on (3). It can be computed with (5) based on the concatenation of the power consumption/injection vectors at any instant and at any node $\mathbf{p}_{t, n}$ and on the voltage at the transformer node at any instant. This expression is similar to (1), the only difference is that (5) computes the concatenation of vectors $\mathbf{v}_{t_{k}, n}$.

Vectors $\mathbf{v}_{t, n}$ and $\mathbf{p}_{t, n}$ contain voltages an powers respectively, organized in blocks classified by instants and then by nodes. For the sake of simplicity of the optimization problem approach, matrix $\hat{\mathbf{A}}$ and vectors $\mathbf{p}_{t, n}$ and $\hat{\mathbf{h}}_{t}$ can be rearranged to get an organization of blocks classified first by nodes and then by instants in order to get a vector of voltages organized in the same way. For nodes $i=\{1,2, \cdots N\}$ and instants $k=\{1,2, \cdots f\}$, the desired voltage vector should have the following structure.

$$
\begin{aligned}
\mathbf{v}_{i, t} & =\left[v_{i, t_{1}}, v_{i, t_{2}}, \cdots, v_{i, t_{f}}\right]^{\mathrm{T}} \\
\mathbf{v}_{n, t}^{\mathrm{T}} & =\left[\mathbf{v}_{1, t}^{\mathrm{T}}, \mathbf{v}_{2, t}^{\mathrm{T}}, \cdots, \mathbf{v}_{N, t}^{\mathrm{T}}\right]
\end{aligned}
$$

This rearrangement provides the matrix $\mathbf{A}$ and vectors $\mathbf{p}_{n, t}$, and $\mathbf{h}_{t}$ which can be employed to compute the desired voltage vector with the following expression.

$$
\mathbf{v}_{n, t}=\mathbf{A} \mathbf{h}_{t}+\mathbf{A} \mathbf{p}_{n, t}
$$

\section{B. State of Charge}

The state of charge for a PHEV corresponding to node $i$ at any time is given by, the following expression.

$$
\mathbf{c}_{i, t}=\hat{\mathbf{B}} \mathbf{p}_{i, t}
$$

Where matrix $\hat{\mathbf{B}}$ is common to each PHEV and it has the following structure.

$$
\hat{\mathbf{B}}=\left[\begin{array}{ccccc}
\Delta t & 0 & & \cdots & 0 \\
\Delta t & \Delta t & & & \vdots \\
\vdots & & \ddots & 0 & 0 \\
\Delta t & \Delta t & \cdots & \Delta t & 0 \\
\bar{\Delta} \bar{t} & \bar{\Delta} t & \cdots & -\overline{\Delta t} & \overline{\Delta t}
\end{array}\right]=\left[\begin{array}{c}
\hat{\mathbf{B}}_{u} \\
\hat{\mathbf{b}}_{d}^{\mathrm{T}}
\end{array}\right]
$$

The state of charge for each PHEV in the grid during the charging period can be separated in two expressions representing the state of charge before the final instant (computed with $\hat{\mathbf{B}}_{u}$ ), and the final state of charge (computed with $\hat{\mathbf{b}}_{d}$ ).

$$
\begin{aligned}
\mathbf{B}_{u}= & {\left[\begin{array}{lll}
\hat{\mathbf{B}}_{u} & & \mathbf{0} \\
& \ddots & \\
\mathbf{0} & & \hat{\mathbf{B}}_{u}
\end{array}\right], \mathbf{B}_{d}=\left[\begin{array}{lll}
\hat{\mathbf{b}}_{d}^{\mathrm{T}} & & \mathbf{0} \\
& \ddots & \\
\mathbf{0} & & \hat{\mathbf{b}}_{d}^{\mathrm{T}}
\end{array}\right] } \\
& \left\{\begin{array}{l}
\mathbf{c}_{n,\left\{t_{1}, \cdots, t_{f}-\Delta t\right\}}=\mathbf{B}_{u} \mathbf{p}_{n, t} \\
\mathbf{c}_{n,\left\{t_{1}, \cdots, t_{f}\right\}}=\mathbf{B}_{d} \mathbf{p}_{n, t}
\end{array}\right.
\end{aligned}
$$




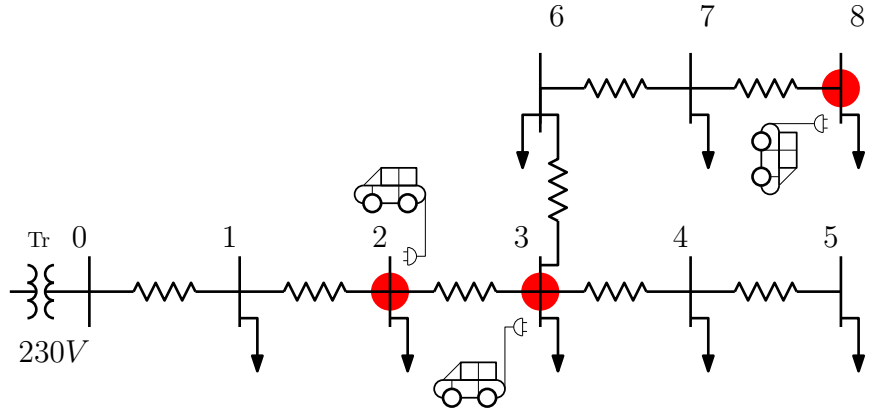

Fig. 2. Radial Grid proposed in order to test the strategy.

Matrix $\mathbf{B}_{u}$ in (7) is constructed with individual matrices $\hat{\mathbf{B}}_{u}$ and is employed to compute the state of charge until the time instant before the end of the charging period for every PHEV in the grid. On the other hand, $\mathbf{B}_{d}$ is constructed with individual transposed vectors $\hat{\mathbf{b}}_{d}$ and is employed to compute the final state of charge as in (8).

\section{RESTRICTIONS FOR THE CHARGING OF PHEVS}

The most important objective of the intelligent charging approach is to reduce the impact on the grid caused by charging the PHEVs. In this case, the approach followed is to provide voltage support to every node in the grid. On the optimization problem, this is imposed as a restriction on the voltages of every node even if there is absence of PHEV. The restrictions for voltage support are summarized as follows.

$$
\left\{\begin{array}{l}
\mathbf{A} \mathbf{p}_{n, t} \leq \mathbf{v}_{\text {max }}-\mathbf{A} \mathbf{h}_{t} \\
\mathbf{A} \mathbf{p}_{n, t} \geq \mathbf{v}_{\text {min }}-\mathbf{A} \mathbf{h}_{t}
\end{array}\right.
$$

Where, $\mathbf{v}_{\max }$ and $\mathbf{v}_{\min }$ are vectors including the maximum and minimum voltages allowed for each node of the grid. By default, these limits are set between $90 \%-110 \%$ of the nominal voltage. It is important to notice that the term $\mathbf{A} \mathbf{h}_{t}$ is independent from the power consumption or injection from PHEVs.

The power injected/consumed by PHEVs is restricted as follows.

$$
\left\{\begin{array}{l}
\mathbf{p}_{n, t} \leq \mathbf{p}_{\max } \\
\mathbf{p}_{n, t} \geq-\mathbf{p}_{\max }
\end{array}\right.
$$

The chargers are assumed reversible. Power injected or consumed by PHEVs is restricted to the maximum input/output power which depends on the type of charger. In order to improve live expectancy of batteries, the state of charge is also restricted as follows.

$$
\left\{\begin{array}{l}
\mathbf{B}_{u} \mathbf{p}_{n, t} \leq \mathbf{c}_{\text {max }_{u}}-\mathbf{c}_{i n i_{u}} \\
\mathbf{B}_{u} \mathbf{p}_{n, t} \geq-\mathbf{c}_{i n i_{u}} \\
\mathbf{B}_{d} \mathbf{p}_{n, t}=\mathbf{c}_{\text {max }_{d}}-\mathbf{c}_{i n i_{d}}
\end{array}\right.
$$

Where, the limits are established between zero and a maximum value $c_{\max }$. Here, the first two restrictions limit the state of charge to be within the established limits during the charging period. The third restriction ensures that the state of

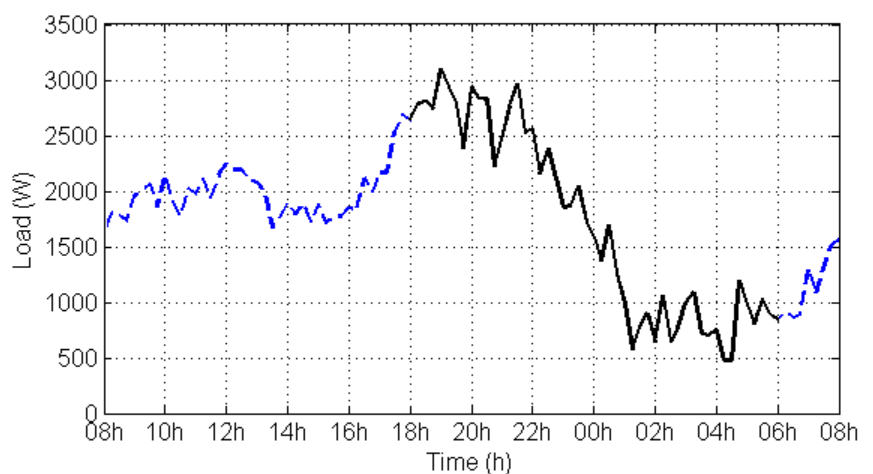

Fig. 3. Sample Load Profile corresponding to a house consumption during 24 hours. The continuous segment corresponds to the chosen charging period for PHEVs plugged in the grid.

charge will be maximum at the end of the charging period. Sub-indexes $u$ and $d$ on the right of the inequalities and the equation, are only placed to specify and match dimensions. The initial states of charge are included in vector $\mathbf{c}_{i n i}$.

\section{Linear Programming APPROACH}

The objective is to minimize the cost of the energy consumed in order to recharge the PHEVs while the restrictions of voltage support, input/output powers, and state of charge are accomplished. The following is the problem stated in standard form, where the powers consumed or injected by the PHEVs are represented in the vector $\mathbf{p}_{n, t}=\mathbf{r}_{n, t}-\mathbf{s}_{n, t}$.

$$
\operatorname{minimize} \Delta t\left[\begin{array}{l:l:l}
\mathbf{k}^{\mathrm{T}},-\mathbf{k}^{\mathrm{T}}, \mathbf{0}
\end{array}\right]\left[\begin{array}{c}
\mathbf{r}_{n, t} \\
\mathbf{s}_{n, t} \\
\mathbf{y}
\end{array}\right]
$$

subject to

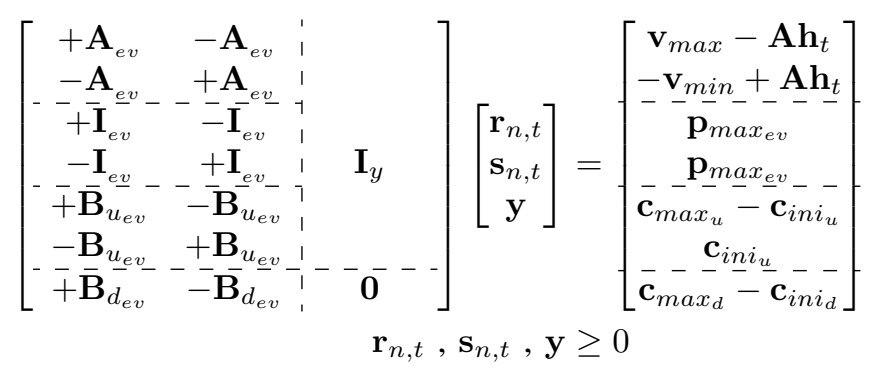

$\mathbf{A}_{e v}$ is the same as $\mathbf{A}$ by eliminating the columns corresponding to nodes without PHEV. $\mathbf{I}_{e v}$ is an identity matrix with dimensions matching the number of columns of $\mathbf{A}_{e v}$. $\mathbf{B}_{u_{e v}}$ is the same as $\mathbf{B}_{u}$ by eliminating the columns and rows corresponding to nodes without PHEV. $\mathbf{B}_{d_{e v}}$ is the same as $\mathbf{B}_{d}$ by eliminating the columns and rows corresponding to nodes without PHEV. In the vector of costs, $\mathbf{k}$ has information of the tariff at each instant of the charging period.

\section{REsults}

A grid model for test is shown on Fig. 2. This is a residential grid with 8 nodes, each corresponding to a house. 3 PHEVs are assumed to be plugged-in during the charging period. They 


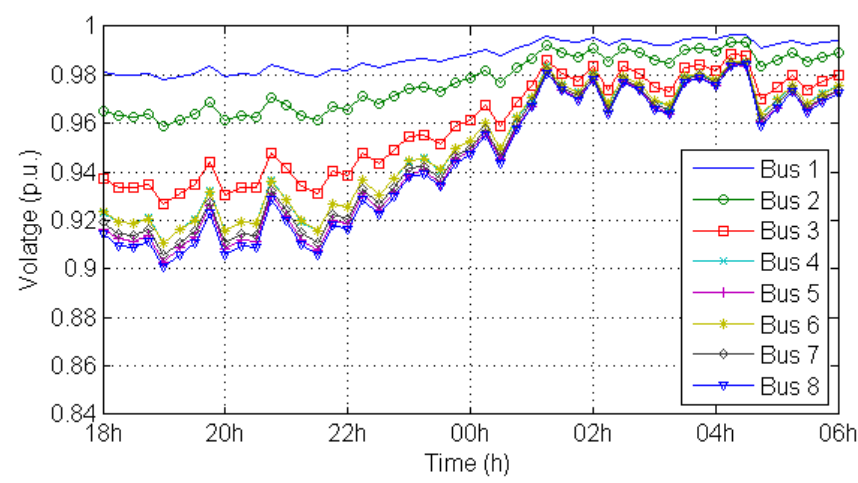

Fig. 4. Voltage Profiles for the test grid case 1 without PHEVs connected. Profiles do not evidence voltage issues.

TABLE I

GRID LINE PARAMETERS

\begin{tabular}{|c|c|c|c|}
\hline \multicolumn{2}{|c|}{ Line } & \multirow[b]{2}{*}{ Case 1} & \multirow[b]{2}{*}{ Case 2} \\
\hline From & To & & \\
\hline 0 & 1 & $0.05 \Omega$ & $0.05 \Omega$ \\
\hline 1 & 2 & $0.05 \Omega$ & $0.05 \Omega$ \\
\hline 2 & 3 & $0.10 \Omega$ & $0.15 \Omega$ \\
\hline 3 & 4 & $0.15 \Omega$ & $0.25 \Omega$ \\
\hline 4 & 5 & $0.15 \Omega$ & $0.15 \Omega$ \\
\hline 3 & 6 & $0.10 \Omega$ & $0.10 \Omega$ \\
\hline 6 & 7 & $0.05 \Omega$ & $0.05 \Omega$ \\
\hline 7 & 8 & $0.10 \Omega$ & $0.10 \Omega$ \\
\hline
\end{tabular}

are connected on the nodes highlighted with a red circle. A sample of a 24 hour load profile is shown on Fig. 3. This load profile is employed as a base for each house on the grid. Additionally, different random numbers (between $-25 \%$ and $25 \%$ of the minimum load in the load profile) are added at each node and at each time. The continuous portion of the profile corresponds to the charging period, between $18 \mathrm{~h}$ and $06 \mathrm{~h}$. The voltage at the transformer node is $230 \mathrm{~V}$. Chargers are restricted to maximum input/output power of $\pm 3 \mathrm{~kW}$. Batteries have a nominal $20 \mathrm{kWh}$ capacity, however only $80 \%$ of the capacity $(16 \mathrm{kWh})$ is employed to reduce the impact on the life expectancy.

The line parameters of the grid are listed in Table I. Two cases are evaluated. On the first case, without considering PHEVs connected to the grid, the load profile does not cause voltage limit violation as it can be seen on Fig. 4. This figure shows the voltage profiles at each node during the charging period, without any PHEV connected. It is possible to see that each voltage profile remains between 0.9p.u. and 1p.u. during this period. The second case has a slight variation on the resistive value of two lines. However, this modification is enough to cause voltage limit violation on certain nodes at certain instants of peak demand, even without PHEVs connected. For this case, the voltage profiles for each node and at each instant (without PHEVs) are shown on Fig. 5.

In this study, the PHEVS are assumed to have a certain initial SOC: We have $10 \%, 5 \%$ and $50 \%$ of the maximum

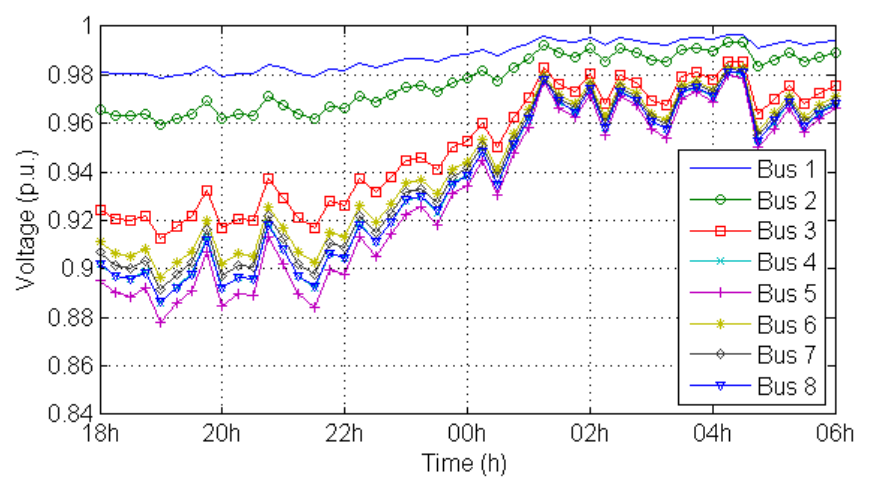

Fig. 5. Voltage Profiles for the test grid case 2 without PHEVs connected. Certain profiles exceed established security limits.

SOC for the first, second, and third PHEV respectively.

\section{A. PHEVs without charging management - Grid Case 1}

When PHEVs are plugged into the grid without any charging management, the chargers are supposed to consume the maximum possible input power in order to charge the batteries as fast as possible. When batteries are fully charged, the corresponding charger cuts out the power consumption. Fig.6(a) shows the power consumption profiles described before. In this scenario, the power consumed to charge the PHEVs ( $3 \mathrm{~kW}$ during $4.5 \mathrm{~h}$ for the first PHEV, 4.75h for the second PHEV and 2.25h for the third PHEV) coincides with the peak hours of power demanded by the houses. Fig.6(b) shows the corresponding SOCs. As it can be seen, batteries are fully charged in a little bit more than a third of the time available for the charging period.

For each node, the resulting voltage profiles can be seen on Fig. 6(c). Comparing with the voltage profiles without PHEVs, shown on Fig. 4, it can be seen that nodes $4-8$ go below the established security limit (0.9p.u.) between $18 \mathrm{~h}$ and $21: 45 \mathrm{~h}$ because of the elevated power consumption. If this conditions are applied to the grid case 2, it is possible to conclude that the PHEVS power consumption will force the voltage profiles drop even more that those of Fig. 5 and Fig. 6(c).

In this case, a unique fictive tariff is considered: $1 \$ / \mathrm{kWh}$. The resulting cost of charging the PHEVs is $37.6 \$$. This is proportional to the total amount of energy required to charge the batteries which is $37.6 \mathrm{kWh}$ (corresponding to $90 \%, 95 \%$, and $50 \%$ of $16 \mathrm{kWh}$ ).

\section{B. Charging management - Grid Case 1 - Unique Tariff}

Applying the optimization strategy to the first grid scenario results in the power consumption profiles of Fig. 7(a). As a result, the power consumption is redistributed during the whole charging period. It is important to highlight that the maximum general consumption occurs during the low demand hours. Also, it is important to notice that during the peak demand hours, the most initially charged PHEV injects energy to the grid in order to compensate the consumption of the other PHEVs and maintain the voltages within the security 


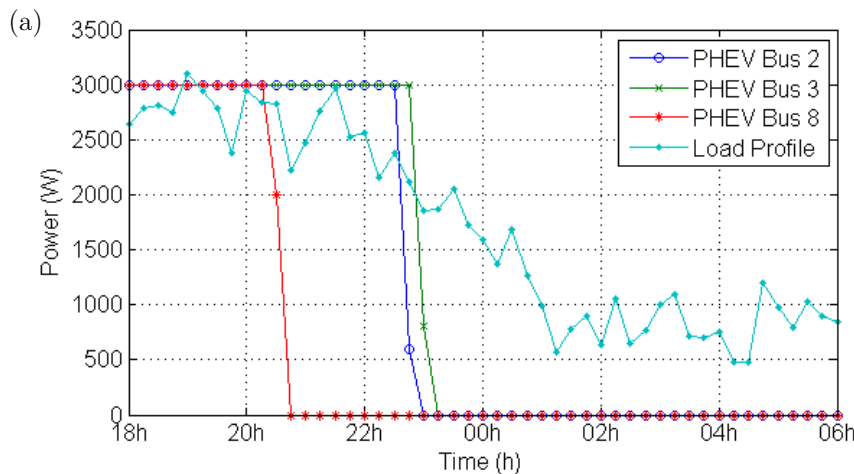

(b)
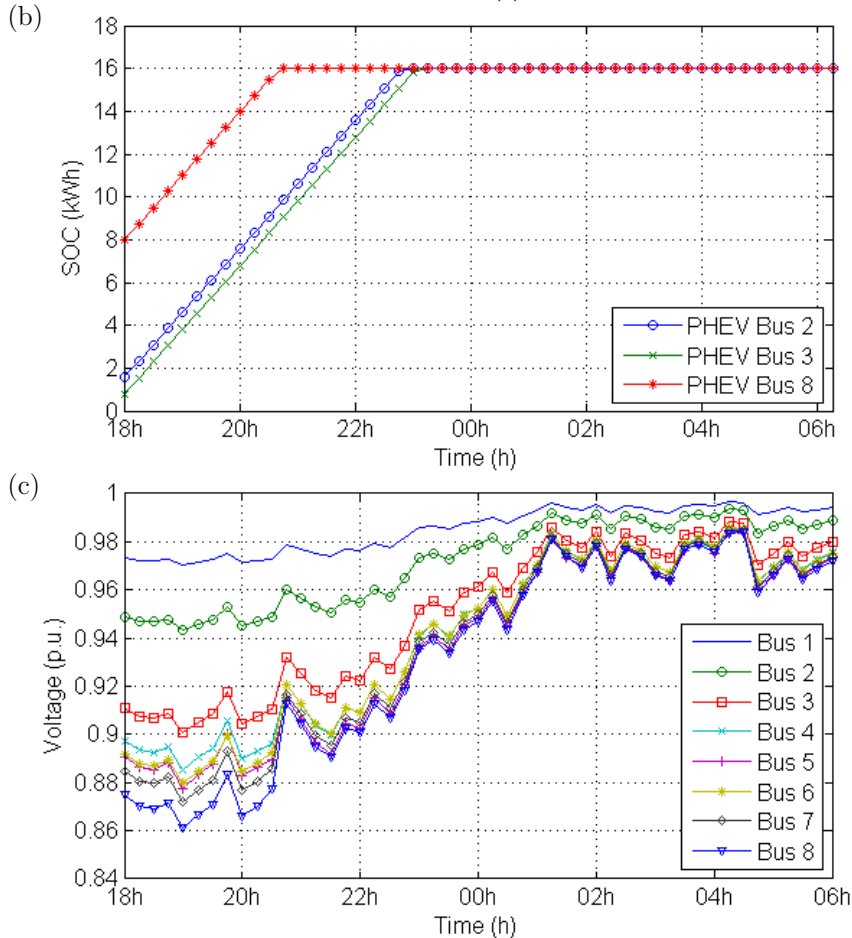

Fig. 6. Power consumption of PHEVs, State of Charge of Batteries, and Voltage Profiles for the grid case 1 without charging management.

limits. The SOC profiles are shown on Fig. 7(b). It can be seen that at the end of the charging period, the batteries are fully charged. The voltage profiles corresponding to every node are kept within the desired limits, even during peak demand hours, as it is shown on Fig. 7(c).

In this case, the same unique fictive tariff is considered: $1 \$ / \mathrm{kWh}$. The optimal cost of charging the PHEVs is also $37.6 \$$ as in the suboptimal case. The cost is the same because the total amount of energy required to fully charge the PHEVs is still $37.6 \mathrm{kWh}$.

\section{Charging management - Grid Case 1 - Two Tariff Scenario}

Now, let us consider two tariffs: $1.5 \$ / \mathrm{kWh}$ during $18 \mathrm{~h}-$ $22 \mathrm{~h}$, and $1 \$ / \mathrm{kWh}$ during $22 \mathrm{~h}-06 \mathrm{~h}$. In the suboptimal case, the consumption is concentrated during the interval of high price. As a consequence, the cost of charging the PHEVs without any management is $53.6 \$$.

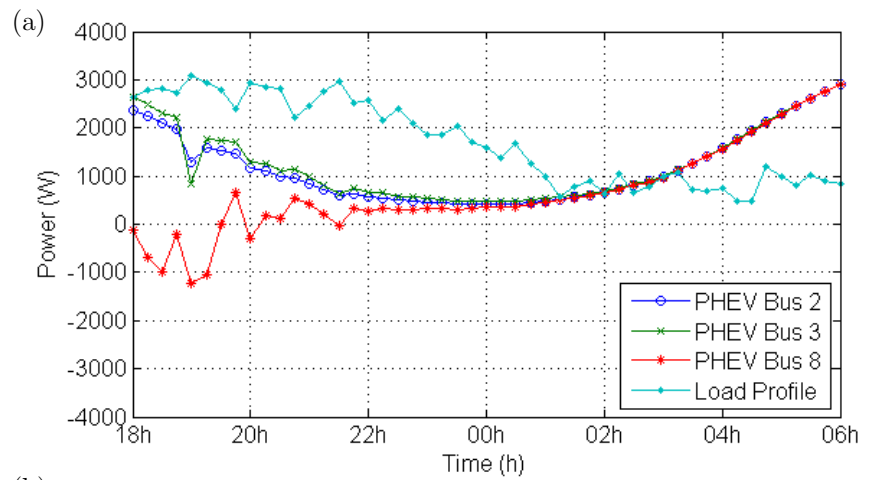

(b)

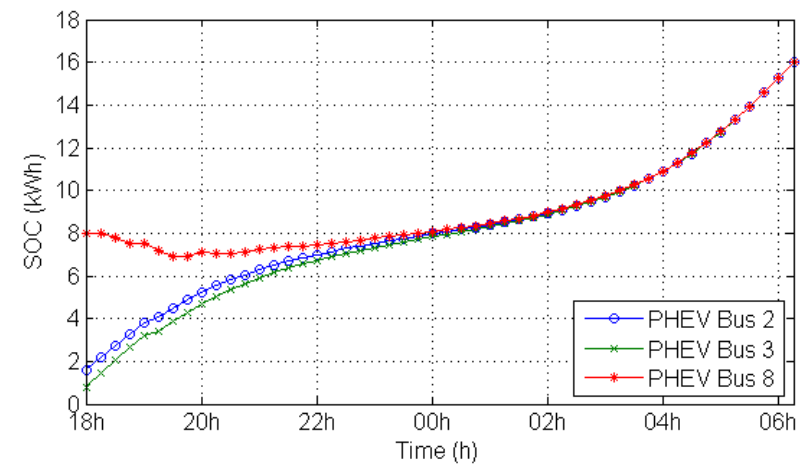

(c)

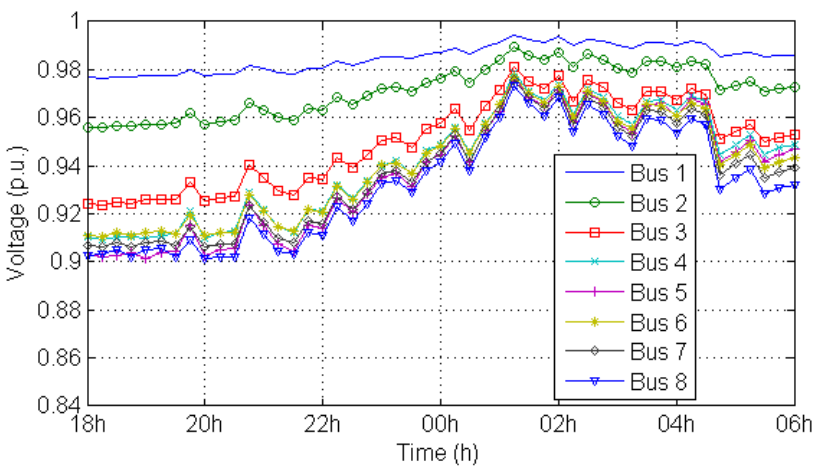

Fig. 7. Power consumption of PHEVs, State of Charge of Batteries, and Voltage Profiles for the grid case 1 with the application of the charging management approach.

Applying the optimization strategy to the two tariff scenario results in the power consumption profiles of Fig. 8(a). The power consumption is also redistributed during the whole charging period. However, the PHEVs now try to sell as much of their initial SOC as possible during the high-price hours, in order to reduce the cost of recharging their batteries. It is possible to confirm this in the SOC profiles of Fig. 8(b). Although the first and second PHEVs charge their batteries at the beginning of the charging period, at the end of the highprice hours the three PHEVs are fully discharged. They sell their initial energy and during the low-price hours, they fully recharge their batteries. As it is required, the voltage profiles corresponding to every node are kept within the desired limits, even during peak demand hours, as it is shown on Fig. 8(c).

The optimal cost of charging the PHEVs is now reduced to $32.4 \$$. This cost corresponds to the cost of fully charging the batteries during the low-price hours $(3 \times 16 \mathrm{kWh} \times 1 \$ / \mathrm{kWh}=$ 


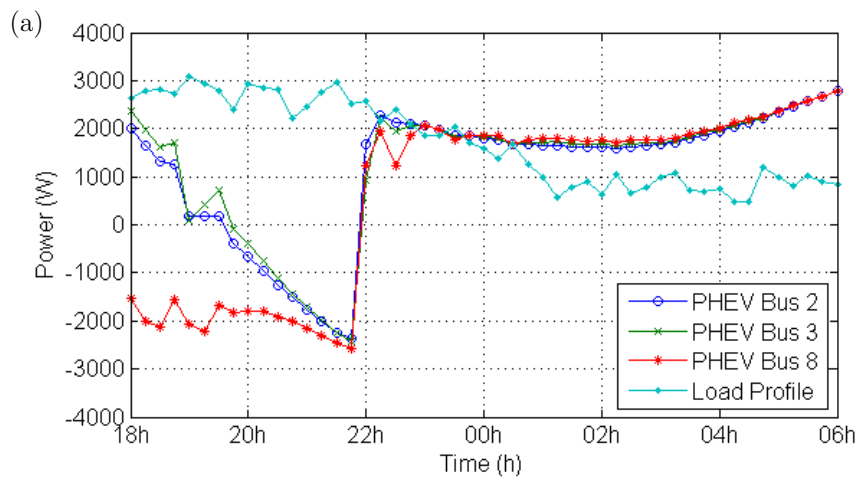

(b)

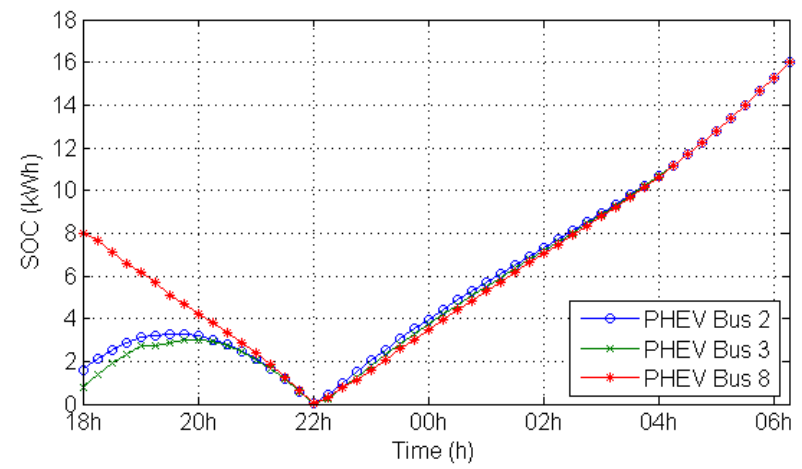

(c)

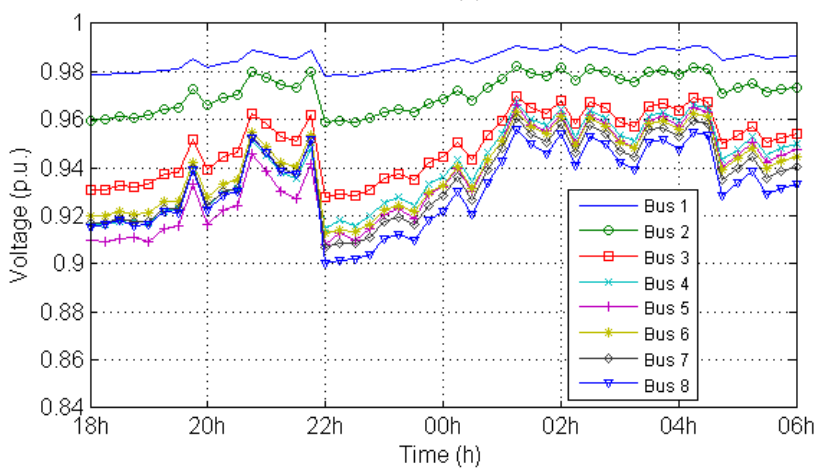

Fig. 8. Power consumption of PHEVs, State of Charge of Batteries, and Voltage Profiles for the grid case 1 with the application of the charging management approach and the inclusion of two tariffs.

$48.0 \$)$ minus the profit obtained from selling the initial energy stored in the batteries during the high-price hours $(16 \mathrm{kWh} \times 0.65 \times 1.5 \$ / \mathrm{kWh}=15.6 \$)$.

\section{Charging management - Grid Case 2}

Assuming again a unique tariff, the grid case 2 is evaluated. In this case, the voltage profiles do not respect the security limits during peak hours, even without PHEVs. Given the fact that the peak hours coincide with the beginning of the charging period, if PHEVs do not have certain initial energy stored in their batteries, the restriction of voltage support cannot be respected and there is no feasible solution. The required initial amount of energy is somehow proportional to the deviation of voltages from the desired limits.

If the initial amount of energy available in the batteries is enough, the PHEVs can provide a voltage support service to the grid by injecting this energy and compensating the

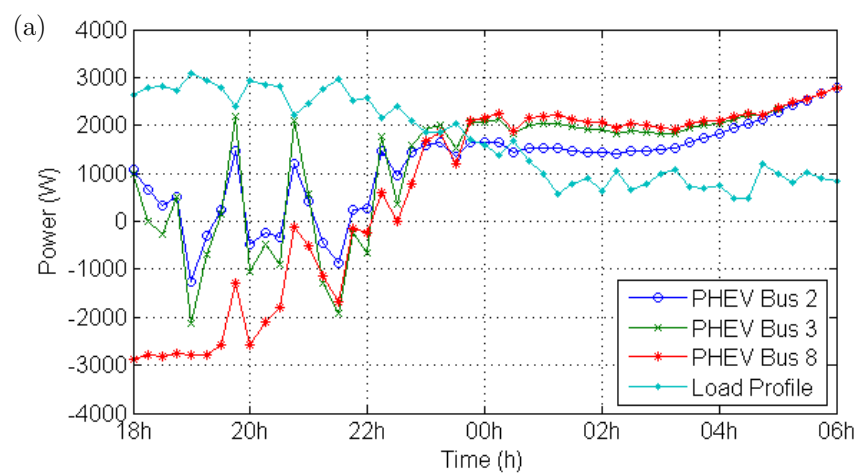

(b)

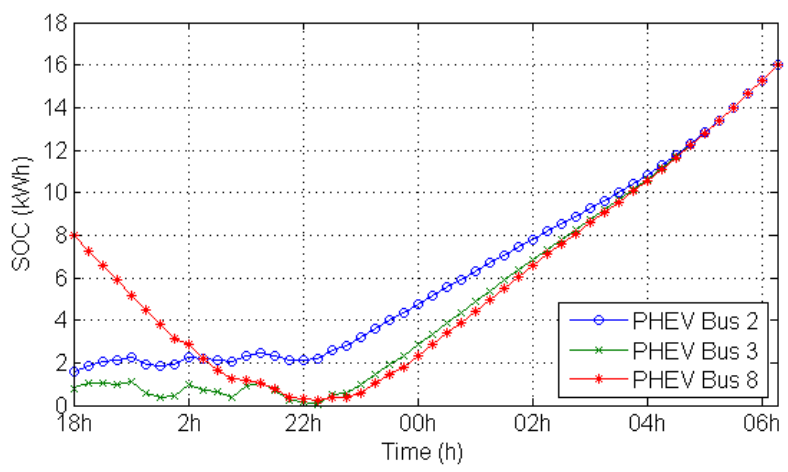

(c)

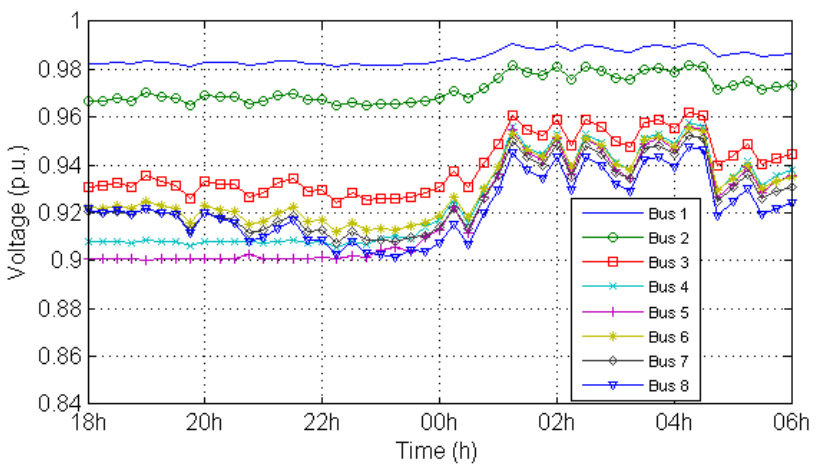

Fig. 9. Power consumption of PHEVs, State of Charge of Batteries, and Voltage Profiles for the grid case 2 with the application of the charging management approach

house load consumption. Fig. 5 shows that without PHEVs, nodes $4-8$ go below the established security limit. Applying the optimization strategy results in the power consumption profiles of Fig. 9(a) and the SOC profiles of Fig. 9(b). Here the most initially charged PHEV (in node 8) injects energy to the grid during peak hours, almost until it is fully discharged. Meanwhile, the other PHEVs charge and discharge their batteries. After $22 \mathrm{~h}$ when the demand begins to decrease, The PHEVS begin to consume energy until they are fully charged. It is important to notice that the initial voltage issue of the grid is solved while the support service is provided to every node on the grid as it can be seen on Fig. 9(c).

In this case, the cost is still $37.6 \$$ as in the case 1 , because the total amount of energy required to fully charge the batteries is still $37.6 \mathrm{kWh}$. 


\section{CONCLUSION}

This paper provides a linear approach to compute the voltages on a residential grid based on the house instantaneous load and the instantaneous consumption/injection of PHEVs. Based on this linear modeling, The paper proposes a linear programming formulation to manage the charging of multiple PHEVs on a residential grid under certain assumptions. Additionally, the proposed strategy employs the energy storage capacity of the PHEVs in order to provide a voltage support service to the residential grid. The proposed scheme is evaluated under multiple cases in order to test its ability to maintain voltages within safety limits and provide optimal consumption/injection policies. Then, the results are analyzed in detail.

Multiple alternative tests can be considered in the future: The inclusion of a differentiation between energy selling prices and buying prices; the analysis under stochastic conditions for the prices or loading profiles, etc.

\section{REFERENCES}

[1] H. Turker, S. Bacha, and D. Chatroux, "Impact of plug-in hybrid electric vehicles (phevs) on the french electric grid," in 2010 IEEE PES Innovative Smart Grid Technologies Conference Europe (ISGT Europe), Oct 2010, pp. $1-8$.

[2] H. Turker, S. Bacha, D. Chatroux, and A. Hably, "Low-voltage transformer loss-of-life assessments for a high penetration of plug-in hybrid electric vehicles (phevs)," IEEE Transactions on Power Delivery, vol. 27, no. 3, pp. 1323-1331, July 2012.

[3] B.-G. Kim, S. Ren, M. van der Schaar, and J.-W. Lee, "Bidirectional energy trading and residential load scheduling with electric vehicles in the smart grid," IEEE Journal on Selected Areas in Communications, vol. 31, no. 7, pp. 1219-1234, July 2013.

[4] H. K. Nguyen and J. B. Song, "Optimal charging and discharging for multiple phevs with demand side management in vehicle-to-building," Journal of Communications and Networks, vol. 14, no. 6, pp. 662-671, Dec 2012.

[5] R. Couillet, S. Perlaza, H. Tembine, and M. Debbah, "Electrical vehicles in the smart grid: A mean field game analysis," IEEE Journal on Selected Areas in Communications, vol. 30, no. 6, pp. 1086-1096, July 2012.

[6] H. Turker, S. Bacha, and A. Hably, "Rule-based charging of plugin electric vehicles (pevs): Impacts on the aging rate of low-voltage transformers," IEEE Transactions on Power Delivery, vol. PP, no. 99, pp. 1-1, 2014.

[7] K. Clement-Nyns, E. Haesen, and J. Driesen, "The impact of charging plug-in hybrid electric vehicles on a residential distribution grid," IEEE Transactions on Power Systems, vol. 25, no. 1, pp. 371-380, Feb 2010.

[8] _ , "The impact of vehicle-to-grid on the distribution grid," Electric Power Systems Research, vol. 81, no. 1, pp. 185 - 192, 2011. 